\title{
A FRAMEWORK TO MANAGE UNCERTAINTY IN THE COMPUTATION OF WASTE COLLECTION ROUTES AFTER A FLOOD
}

\author{
Arnaud Le Guilcher ${ }^{\mathrm{a}, *}$, Sofiane Martel ${ }^{\mathrm{a}}$, Mickaël Brasebin ${ }^{\mathrm{a}, \mathrm{b}}$, Yann Méneroux ${ }^{\mathrm{a}}$ \\ ${ }^{a}$ LASTIG, Univ Gustave Eiffel, ENSG, IGN, F-94160 Saint-Mande, France \\ arnaud.le-guilcher@ign.fr \\ ${ }^{\mathrm{b}}$ AGATE-Agence Alpine des Territoires, Chambéry, France
}

Commission IV, WG IV/3

KEY WORDS: Vehicle Routing, Stochastic Optimization, Uncertainty, Meta-heuristics, Disaster Preparedness.

\begin{abstract}
:
In this paper, we describe a framework to find a good quality waste collection tour after a flood, without having to solve a complicated optimization problem from scratch in limited time. We model the computation of a waste collection tour as a capacitated routing problem, on the vertices or on the edges of a graph, with uncertain waste quantities and uncertain road availability. Multiple models have been conceived to manage uncertainty in routing problems, and we build on the ideas of discretizing the uncertain parameters and computing master solutions that can be adapted to propose an original method to compute efficient solutions. We first introduce our model for the progressive removal of the uncertainty, then outline our method to compute solutions: our method first considers a low-dimensional set of random variables that govern the behaviour of the problem parameters, discretizes these variables and computes a solution for each discrete point before the flood, and then uses these solutions as a basis to build operational solutions when there are enough information about the parameters of the routing problem. We then give computational tools to implement this method. We give a framework to compute the basis of solutions in an efficient way, by computing all the solutions simultaneously and sharing information (that can lead to good quality solutions) between the different problems based on how close their parameters are, and we also describe how real solutions can be derived from this basis. Our main contributions are our model for the progressive removal of uncertainty, our multi-step method to compute efficient solutions, and our intrusive framework to compute solutions on the discrete grid of parameters.
\end{abstract}

\section{INTRODUCTION}

The managing of risks associated with floods is a vast and important research domain. After a flood or another disaster, it is crucial for cities to recover quickly and be able to run the most vital activities as soon and as well as possible. In this endeavour, the clearing of unpracticable roads is a major stage, and that often involves the collection of waste created by the flood. Efficient waste collection is thus a major component for the resilience of territories (Beraud, 2013). These waste include vegetation, construction materials, domestic waste, household appliances, individual transportation vehicles, dead animals, chemical products... Their quantity can amount to several years worth of domestic waste in normal conditions. For example, the volume of waste produced by hurricane Katrina in 2005 is estimated to be around 90 millions of cubic meters (Luther, 2010). Immediately after a natural catastrophe, the first goal is to clear roads by pushing waste on the side of the road, to facilitate urgent operations (for health, security...). Once these most pressing activities are over, waste are collected and taken to specific facilities. To set up efficient waste collection tours, it would be useful to know waste quantities beforehand, but these quantities are very hard to evaluate. Methods have been proposed to quantify domestic waste, using census data (Beraud et al., 2012), but they can not be very precise, and waste generated by floods remain inherently hard to predict. It is therefore important to take into account this high level of uncertainty when setting up waste collection tours.

The management of post-disaster waste has been studied intens-

${ }^{*}$ Corresponding author ively in the literature (Brown et al., 2011), while the problem of the collection of regular waste has also been treated with a high number of different methods (Bányai et al., 2019). The more specific study of the logistics of post-disaster waste collection has given rise to three main problems. The location problem consists in finding appropriate locations for depots (where waste will be gathered) (Owen and Daskin, 1998), (Mladenović et al., 2007). The allocation problem must establish a correspondance between depots and demand sites, where waste lies before being taken to depots. The goal of the routing problem is to schedule routes for a fleet of vehicle in order to pick up the waste at demand sites and bring it to the depot site. The routing problem can be modelled by a wide variety of optimization problems, depending on the constraints that are implemented and the hypotheses on the location of waste. The specific problem of waste collection after a disaster is often modelled as a capacitated vertices routing problem, or as a capacitated arc routing problem. These problems and the various resolution methods are described in (Laporte, 1992) and (Wøhlk, 2008). Many resolution methods have been tested on these routing problems. Exact methods can use a quasi-comprehensive exploration of the space of solutions with branch and cut methods, or dynamic programming (Yi and Özdamar, 2007). Some authors insist on the importance of computing good approximate solutions quickly, even at the expense of optimality (Çelik et al., 2015). Approximate methods generally use problem-specific heuristics or meta-heuristics. Among the meta-heuristics used to solve routing problems, one can cite simulated annealing, ant colonies, genetic algorithms, or tabu search (Shen et al., 2009). Works that take into account the uncertainties in the parameters of the location problem or the routing problem are 
fewer. Some of them manage the uncertainty by establishing a small list of scenarios, and look for solutions that perform the best across these scenarios, when some decisions (typically the location of depot sites) have to be taken in advance (Mete and Zabinsky, 2010) (Rawls and Turnquist, 2010). Others adopt a more continuous approach and study the behaviour of solutions when some events are assigned probabilities (Campbell and Jones, 2011), or combine a meta-heuristic search with Monte-Carlo simulation to assess the performance of solutions across the range of possible values for parameters of the problem (Gruler et al., 2017). Routing problems with stochastic demand have been studied since (Jaillet, 1985), (Bertsimas et al., 1988) and (Jaillet, 1988), and the aim of the research on this subject has been to find a priori solutions that can lead to the derivation of good solutions for all realizations of the stochastic variables. This adaptation generally takes the form of simple recourse actions to include new points or ensure the capacities of vehicles are not exceeded. Oyola et al. (Oyola et al., 2018) give an overview of probability distributions and recourse actions put forward in the literature. Sampling scenarios and combining their solutions has also been used to solve stochastic routing problems (Hvattum et al., 2006). Robust optimization (Ben-Tal and Nemirovski, 1998), (El Ghaoui et al., 1998) has been put forward, for problems with stochastic parameters, to find solutions that have good performance even in the worst-case scenarios, by setting a min-max objective. The Master and Daily Scheduling model (Sungur et al., 2010) uses several of these ideas to compute a master solution that can be transformed into good quality solutions for a range of instances. Daily schedules derived from this master solution are then improved by tabu search.

The uncertainty that has to be accounted for in the calculation of efficient waste collection tours has two important characteristics:

- It is very high-dimensional. After a flood, every single variable, be it quantities of waste along a given street, or even the availability of the street for motorized travel, is uncertain. Models can postulate correlations between the laws of these variables, but they may still take wide ranges of values independently from each other.

- It is removed gradually in time. At first, one only has access to a global evaluation of water height and waste quantities. More precise evaluations may be gained before setting up waste collection tours, but adjustments may still need to be made on the fly, depending on the local situation one vehicle is confronted to.

A high-dimensional uncertainty means that one can not attempt to compute an optimal solution for every single situation that could arise. On the other hand, the gradual removal of the uncertainty incites one to try and compute rough solutions depending on a few high-level, global variables, and refine these master solutions to adapt to the precise situation at hand. In this paper, we will describe a framework on how to manage this gradual removal of the uncertainty, and the computations that need to be done at each stage, and the kinds of solutions that will be sought after.

\section{MODELIZATION OF WASTE COLLECTION TOURS AND REMOVAL OF UNCERTAINTY}

\subsection{Possible models for waste collection tours}

Waste collection tours can be treated with different models. We describe two such models, the choice of which of these models to use depends on the hypotheses one makes on how waste is put down after the flood. If we make the hypothesis that waste is grouped in heaps, for example at nodes of the road network, then one should compute waste collection tours as solutions of a vertices routing problem, where every vertex with a positive quantity of waste must be visited by a vehicle (and waste must be picked up). The capacitated vertices routing problem (CVRP) adds a constraint of vehicle capacity for a more realistic framing of the problem. As the important quantity of waste is one of the key points that make waste collection a complicated task after a flood, we think it is clearly the most useful constraint to add in this context.

We consider a graph $G=(V, E)$, where each vertex $V$ has an associated quantity of waste $q_{V}$, each edge $e$ has a cost $c_{e}$, and we note $Q$ the capacity of a vehicle. One vertex $V_{0}$ is the depot vertex, where each vehicle starts and drops the waste once it has been collected. We introduce the following formulation of the CVRP to mirror the one we give of the CARP below. This formulation is not the simplest, but it gives a very clear idea of the individual vehicle rotations. Some resolution methods may need to work on other format of solutions, but these can easily be deduced from this one, which gives the detailed routes of each rotation.

We look for a collection tour $T=\left(T^{1}, \ldots, T^{N}\right)$ composed of several individual vehicle rotations, where each rotation $T^{k}=$ $\left(X^{k}, Y^{k}\right)$ of length $l_{k}$ contains a path $X^{k}=\left(x_{1}^{k}, \ldots, x_{l_{k}}^{k}\right) \in$ $V^{l_{k}}$ and a vector $Y^{k}=\left(y_{1}^{k}, \ldots, y_{l_{k}}^{k}\right) \in\left(\{0 ; 1\}^{l_{k}}\right.$ that indicates at which vertices the vehicle effectively picks up the waste. The solution must satisfy the following constraints:

- Each individual rotation starts and ends at the depot

$$
\forall 1 \leq k \leq N, x_{1}^{k}=x_{l_{k}}^{k}=V_{O}
$$

- Each path is continuous

$$
\forall 1 \leq k \leq N, \forall 1 \leq i \leq l_{k}-1,\left(x_{i}^{k}, x_{i+1}^{k}\right) \in E
$$

- Each vehicle does not exceed its capacity

$$
\forall 1 \leq k \leq N, \sum_{i=1}^{l_{k}} y_{i}^{k} q_{x_{i}^{k}} \leq Q
$$

- All the waste must be picked up

$$
\begin{gathered}
\sum_{k=1}^{N} \sum_{i=1}^{l_{k}} y_{i}^{k}=\left|\left\{v \in V \mid q_{v}>0\right\}\right| \\
\forall(k, i) \text { with } y_{i}^{k}=1, q_{x_{i}^{k}}>0 \\
\forall\left(k_{1}, i_{1}\right) \neq\left(k_{2}, i_{2}\right) \text { with } y_{i_{1}}^{k_{1}}=y_{i_{2}}^{k_{2}}=1, x_{i_{1}}^{k_{1}} \neq x_{i_{2}}^{k_{2}}
\end{gathered}
$$

The solution must minimize the total cost of the edges that are driven through: 


$$
C=\sum_{k} \sum_{i=1}^{l_{k}-1} c_{\left(x_{i}^{k}, x_{i+1}^{k}\right)}
$$

Another realistic hypothesis for the location of waste is that waste is laid down along the streets. This hypothesis leads to the formulation of another optimization problem, the capacitated arc routing problem (CARP), where all edges with positive quantity of waste must be driven through (and waste must be picked up). This problem can be modelled in a similar way. Here, waste quantities $q_{e}$ are associated with edges of the graph. We give a formulation inspired from (Mei et al., 2010), which has the advantage of detailing each individual route. The solution is a set of individual rotations $\left(T^{1}, \ldots, T^{N}\right)$ with $T^{k}=\left(X^{k}, Y^{k}\right)$. As before, $X^{k}=\left(x_{1}^{k}, \ldots, x_{l_{k}}^{k}\right)$ a tuple of vertices. This time we have $Y^{k}=\left(y_{1}^{k}, \ldots, y_{l_{k}-1}^{k}\right) \in\{0 ; 1\}^{l_{k}-1}$, as the $y_{i}^{k}$ indicate on which edges (not vertices) waste is picked up.

The constraints are written in a similar manner:

$$
\begin{gathered}
\forall 1 \leq k \leq N, x_{1}^{k}=x_{l_{k}}^{k}=V_{O} \\
\forall 1 \leq k \leq N, \forall 1 \leq i \leq l_{k}-1,\left(x_{i}^{k}, x_{i+1}^{k}\right) \in E \\
\forall 1 \leq k \leq N, \sum_{i=1}^{l_{k}-1} y_{i}^{k} q_{\left(x_{i}^{k}, x_{i+1}^{k}\right)} \leq Q \\
\sum_{k=1}^{N} \sum_{i=1}^{l_{k}} y_{i}^{k}=\left|\left\{e \in E \mid q_{e}>0\right\}\right| \\
\forall(k, i) \text { with } y_{i}^{k}=1, q_{\left(x_{i}^{k}, x_{i+1}^{k}\right)}^{k}>0 \\
\forall\left(k_{1}, i_{1}\right) \neq\left(k_{2}, i_{2}\right) \text { with } y_{i_{1}}^{k_{1}}=y_{i_{2}}^{k_{2}}=1, \\
\left(x_{i_{1}}^{k_{1}}, x_{i_{1}+1}^{k_{1}}\right) \neq\left(x_{i_{2}}^{k_{2}}, x_{i_{2}+1}^{k_{2}}\right) \text { and } \\
\left(x_{i_{1}}^{k_{1}}, x_{i_{1}+1}^{k_{1}}\right) \neq\left(x_{i_{2}+1}^{k_{2}}, x_{i_{2}}^{k_{2}}\right)
\end{gathered}
$$

The cost that must be minimized has the same expression as before.

\subsection{A model for the progressive removal of the uncertainty}

The uncertainties we want to take into account in the computation of efficient waste collection tours are of two types:

- The uncertainties on the quantities of waste $q_{V}$ (or $q_{e}$ depending on the model)

- The uncertainties on the availability of individual streets for motorized travelling. We write $\rho_{e}=1$ when a given edge is available, $\rho_{e}=0$ when it is not.

Waste quantities will probably grow between the first hours after the flood and the time waste is collected, and conversely the availability of roads may get better. As we need to evaluate the values of these uncertain quantities at the time the first response waste collection tour will be carried through, we do not model their evolution over time (in particular, we consider they do not change too much for the duration of the collection tour). As a consequence, the quantities of waste, their locations, and the set of available streets are fixed for our problem (but unknown at first).

The uncertain waste quantities have probability density functions $f_{V}: \mathbf{R}^{+} \rightarrow[0,1]$ (or $f_{e}: \mathbf{R}^{+} \rightarrow[0,1]$ ), and edges have an availability probability $p_{e}=\mathbf{P}\left[\rho_{e}=1\right]$. Little is known of the probabilities before knowing the specifics of the flood. Moreover, information about the severity of the flood, the quantities of waste and the availability of individual streets is generally not known all at once but progressively. Thus, it maked sense to model the progressive removal of uncertainty with a rough filtration

$$
\mathcal{F}_{1} \subset \mathcal{F}_{2} \subset \mathcal{F}_{3}
$$

We do not use a continuous filtration because we try to indentify critical points at which key new information can be obtained. We now describe how the aforementioned probabilities are treated at each stage of the process, and how efficient collection tours can be progressively computed.

- Time $t_{1}$ is during the flood or just after it. At that time, only the global severity of the flood (which can me modelled by a height $h$ ), and a few global indicators concerning waste quantities (which can be given by pre-existing studies, and depend on the severity of the flood) are known. This information is modelled as a vector $\left(h, \xi_{1}, \ldots, \xi_{D}\right)$, where $D$ should remain small.F or example, the $\xi_{i}$ could be the quantities of waste that can potentially be produced by households across different socio-professional categories ; in this case, the quantity of waste expected on a given street depends on these variables and on the population of the street. These $\xi_{i}$ can also be the output of processes, such as a Karhunen-Loève decomposition, designed to produce uncorrelated variables ; in this case, each individual variable $x_{i}$ is less interpretable.

At this stage, each uncertain quantity can be expressed in terms of these variables:

$$
\begin{gathered}
f_{V}\left(\cdot \mid \mathcal{F}_{1}\right)=f_{V}\left(\cdot \mid\left(h, \xi_{1}, \ldots, \xi_{D}\right)\right) \\
p_{e \mid \mathcal{F}_{1}}=p_{e \mid h}
\end{gathered}
$$

We also have access to conditional expectations for the waste quantities:

$$
\mathbf{E}\left[q_{V} \mid \mathcal{F}_{1}\right]=\mathbf{E}\left[q_{V} \mid\left(h, \xi_{1}, \ldots, \xi_{D}\right)\right]
$$

Given this relatively low-dimensional information, an optimal collection tour can be computed for this level of information. As the uncertainty remains high, one may also want to compute several different robust solution, to have the opportunity to adapt one of them into an effective actual solutions at a later time.

- Time $t_{2}$ is the time the collection tour is established. At that time, people that decide on these tours can have access to more local data, and we can expect actual waste quantities not to differ much from their estimations $\mathbf{E}\left[q_{V} \mid \mathcal{F}_{\epsilon}\right]$. With enough local information about the severity of the flood, local road availability should also be known with 
reasonable certainty on the biggest axes. Punctual surprises, such a given waste quantity $q_{V}$ differing greatly from its estimation, or a street being unpracticable even as it was not supposed to be, can still not be excluded.

When this information becomes available, one should be able to compute an efficient tour quickly by adapting one of the solutions computed at time $t_{1}$

- Time $t_{3}$ is the time the waste is collected. At that time, all uncertainty is removed and each quantity is known. Drivers are not expected to make major modifications on the established collection tour, but they might have to adapt if the total quantity at the vertices (or edges) they are expected to service exceeds their capacity, or if a street on their expected path is not available. Then, there have to be guidelines to make these modifications as simple and efficient as possible.

One must note that the solution that is actually carried will generally be computed as a modification of a precomputed solution. As such, it is not the result of a global optimization algorithm, and there is no certainty that it will be the best solution for the actual realization of the waste collection problem. The goal of our method is to compute a final solution that is efficient enough, and the role of the model for the removal of uncertainty and the precomputation of a basis of solutions is to ensure that it will be possible to compute such an efficient solution relatively quickly when it is needed.

\section{METHODS FOR A HIERACHICAL COMPUTATION OF SOLUTIONS}

\subsection{Discretization of the low-dimensional uncertain vari- ables}

We make the hypothesis that the first information that can be gathered about the severity of the flood and the waste quantities is relatively low-dimensional, and described by a vector $\left(h, \xi_{1}, \ldots, \xi_{D}\right)$. As a consequence, it makes sense to precompute a comprehensive set of solutions that can be used as bases for the actual solutions when collection tours are computed.

To compute this base of solutions, the method we propose is to discretize the uncertain variables $h$ and $\xi_{i}$, to obtain a grid for the random variables, as it has been done for partial differential equations(PDEs) since (Ghanem, 1999). Keese (Keese, 2003) gives a comprehensive overview on how such random variables are chosen and discretized, and how PDEs with stochastic coefficient can be solved using this discretization. This kind of discretization has been used for elliptic and parabolic PDEs (Nobile and Tempone, 2009), where solutions generally depend continuously on the random variables, and also for hyperbolic equations (Tryoen et al., 2010), where solutions $u(\cdot, h, \xi)$ can exhibit discontinuities in the physical space but also in the space of random variables.

As the vector $(h, \xi)$ is in $\mathcal{F}_{1}$ and does not resolve all the uncertainty of the collection problem, the quantities $q_{V}$ (or $q_{e}$ ) and $\rho_{e}$ do not have fixed values for a given realization of $(h, \xi)$ (as is usually the case when this discretization framework is used for PDEs), but are defined by their conditional probability density functions or their conditional laws. As we want to compute solutions for set values of these quantities, a natural method to obtain such set values is to consider the expectations for waste quantities, and the most probable result for street availability:

$$
q_{V, \mathcal{F}_{1}}=\mathbf{E}\left[q_{V} \mid \mathcal{F}_{1}\right]
$$

$$
\rho_{e, \mathcal{F}_{1}}=\left\{\begin{array}{l}
0 \text { if } \mathbf{P}\left[\rho_{e}=1 \mid \mathcal{F}_{1}\right]<0.5 \\
1 \text { if } \mathbf{P}\left[\rho_{e}=1 \mid \mathcal{F}_{1}\right] \geq 0.5
\end{array}\right.
$$

To maximize the usefulness of this set of precomputed solutions, every uncertain variable ( $h$ or $\xi_{i}$ ) is discretized according to its prior probability density function. For example, for a variable $\xi$ with prior probability density function

$$
f_{\xi_{i}}: \mathbf{R} \rightarrow[0,1]
$$

$n$ discretization points $\xi_{i}^{(1)}, \ldots, \xi_{i}^{(n)}$ are chosen such that

$$
\int_{-\infty}^{\xi_{i}^{(1)}} f_{\xi_{i}}(t) d t=\int_{\xi_{i}^{(n)}}^{+\infty} f_{\xi_{i}}(t) d t=\frac{1}{n+1}
$$

and for all $1 \leq j \leq n-1$,

$$
\int_{\xi_{i}^{(j)}}^{\xi_{i}^{(j+1)}} f_{\xi_{i}}(t) d t=\frac{1}{n+1}
$$

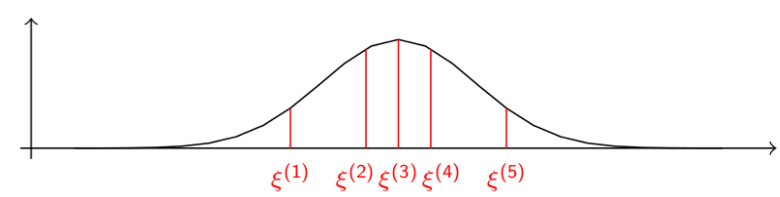

Figure 1. Discretization of an uncertain variable.

This discretization according to prior probability density function is illustrated in figure 1 .

When this discretization is done for every variable, we have a grid on the space of random variables on which we want to compute our base of solutions. Figure 2 illustrates such a grid with two uncertain variables $\xi_{1}$ and $\xi_{2}$.

\subsection{Pre-calculation of robust solutions}

If we consider a low-dimensional vector $(h, \xi)$ (with $D=3$ or 4 for example) and take a small number of discretization points (typically not exceeding 10), it is not unreasonable to try and produce one or a few solutions for every discretized value of the vector $(h, \xi)$, as these computations can be made offline (that is, not during or immediately after a flood, when the need for a quick recovery does not allow the launching of long computations), and once and for all (at least as long as the populations of each individual street do not change too much, which would modify the relationships between the variables $q_{V}$ and $r h o_{e}$ and the vector $(h, \xi)$.

Finding optimal solutions for the CVRP or the CARP can be done using meta-heuristic methods, such as simulated annealing, genetic algorithms, or ant colonies, although exact methods can also be successful even on large problems. While it is possible to compute the solution of each problem independently, 


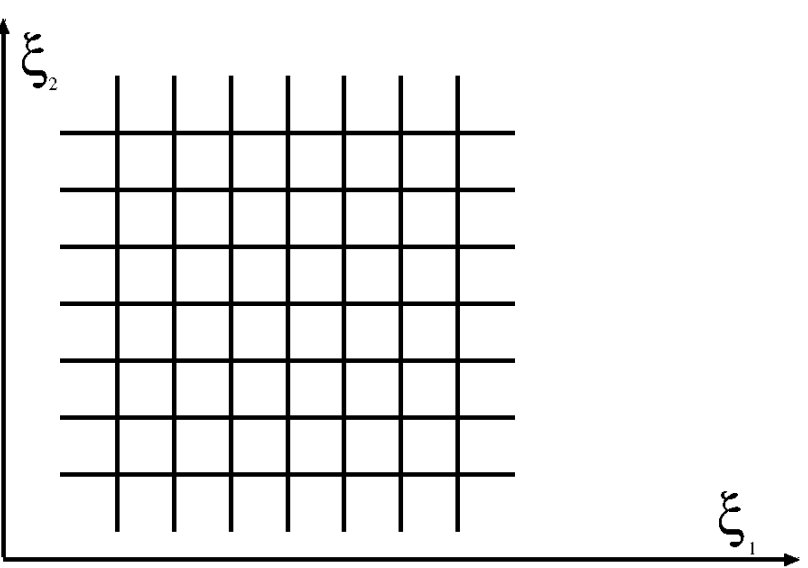

Figure 2. A two-dimensional grid in the space of random variables.

we remark that the routing problems for neighbouring points of the grid in the space of random variables give routing problems whose parameters (waste quantities and road availability) are not very far from each other. Therefore, it maked sense to try and take into account these similarities, and to compute all the solutions simultaneously, while sharing elements between neighbouring nodes at various stages of the computation. This principle can be implemented with algorithms based on various meta-heuristics, where the elements that will be shared will generally be either good solutions for one node, or parameters that lead to the finding of good solutions.

We can give a more precise idea of how this sharing can be done in practice for the most well-known meta-heuristics (ant colonies, simulated annealing, genetic algorithm). Each of these methods functions with discrete steps, where at each step a solution or a group of solutions is computed, using information from the previous step. When solutions are computed simultaneously on the whole grid, at each point $\Xi^{\left(i^{*}, j^{*}\right)}=$ $\left(h^{\left(i^{*}\right)}, \xi_{1}^{\left(j_{1}^{*}\right)}, \ldots, \xi_{D}^{\left(j_{D}^{*}\right)}\right)$, the new solution of group of solutions is dervied using information at the previous step at point $\Xi^{\left(i^{*}, j^{*}\right)}$, and also at neighbouring points $\Xi^{(i, j)}$ with $\left|(i, j)-\left(i^{*}, j^{*}\right)\right|=1$. This communication of information between points of the grid is illustrated by figure 3 .

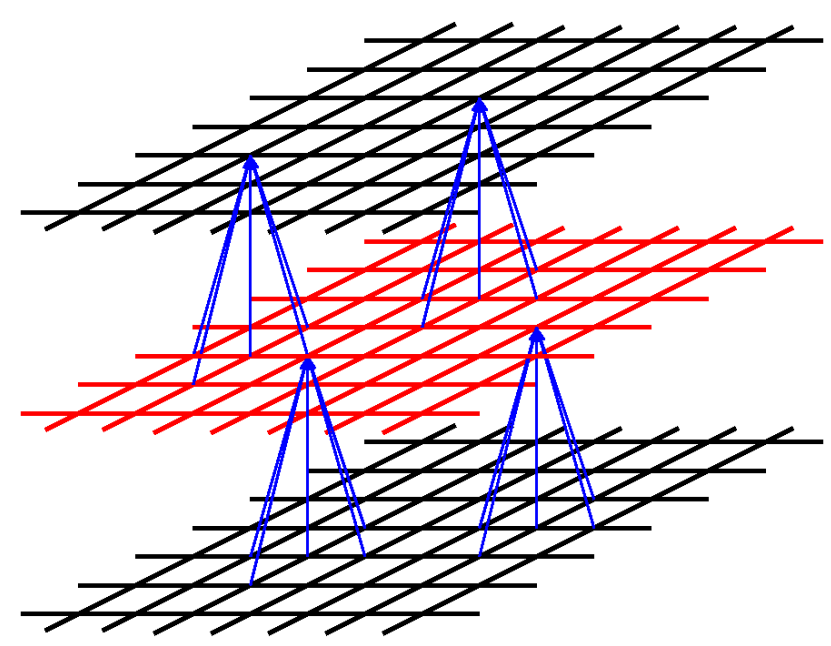

Figure 3. Propagation of information across the grid in the random space.
We give more details for each of these three meta-heuristic methods. for all three methods, the communication of information between nodes would be straightforward, were it not for the changes in the set of available roads, and the sets of available nodes or edges that must be served. Specific adaptations must be made to account for these changes. The guidelines we give are for the CVRP, there are some differences for the CARP, due to the fact that almost all edges must be served.

- Ant colonies : at each point and at each step, a family of solutions is evaluated and, as a result, arcs are given a desirability value (quantity of pheromones in the ant colony analogy) depending on how often they appear on good quality solutions. Here, a simple way to propagate information is to compute these quantities normally for each point of the grid $\Xi^{(i, j)}$, and then replace them by the weighted mean of their values on point $\left(\Xi^{(i, j)}\right)$ and on its neighbours. the weight can be chosen to give more or less importance to the information gathered at the neighbouring points.

If a given edge is not available at one or several of the neighbouring nodes, the mean weighted value at this edge can be taken by excluding these nodes for the computation of the mean, or by including them with the value 0 (to discourage the use of this edge in solutions as its availability is unsure).

- Simulated annealing : We consider a simulated annealing where a solution is an ordered list of vertices. The individual rotations are computed by splitting this solution. For each point, there is one current solution that evolves and converges to an optimal (or a satisfying) solution. At each step, a modified solution is computed. The new current solution is either the better of the former current solution and the modified solution, or the modified solution, this second case being chosen with a probability that diminishes at each step. For a standard simulated annealing computation, the modified solution is created by modification of the current solution. Our proposition to share information between nodes is to take the modified solution at node $\Xi^{(i, j)}$ either as a modification of the current solution at node $\Xi^{(i, j)}$, or as a modification of the current solution at a neighbouring node.

This transmission of information could possibly be made faster if computations are organized such that each step benefits the most from its neighbours information before launching its computation at one step. We can imagine multiple ways to do so, two simple ones are an alternated ordering and a sweeping ordering. In an alternated ordering, at a given step $t \in \mathbf{N}$, only computations for node $\mathrm{s}$ $\Xi^{(i, j)}$ with $i+j$ having the same parity as $n$ are launched. For example, for an even value of $t$, at some node $\Xi^{(i, j)}$ with $i+j$ even, the computation is launched using the state of the solution for node $\Xi^{(i, j)}$ after step $t-2$ as a basis, and using the solutions of neighbouring nodes after step $t-1$. This effectively creates two batches of nodes on which solutions are computed alternately (see figure 4).

For the sweeping ordering, we proceed by alternately sweeping the grid following the direction of each variable. For a sweep in the direction of the variable $\xi_{d}$, one step is performed first for nodes with $j_{d}=1$ (i.e. $\xi_{d}=\xi_{d}^{(1)}$ ), then for nodes with $j_{d}=2$, until we reach the end of the grid with the nodes having $j_{d}=N$ (see figure 5). A sweep in direction $\xi_{d}$ allows a quick transmission of information 


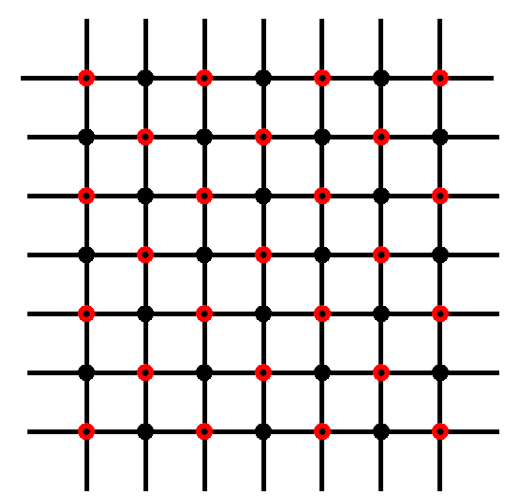

Figure 4. Illustration of the two batches of nodes for the alternated ordering.

in this direction, and alternating these sweeps ensures information is transmitted from all directions.

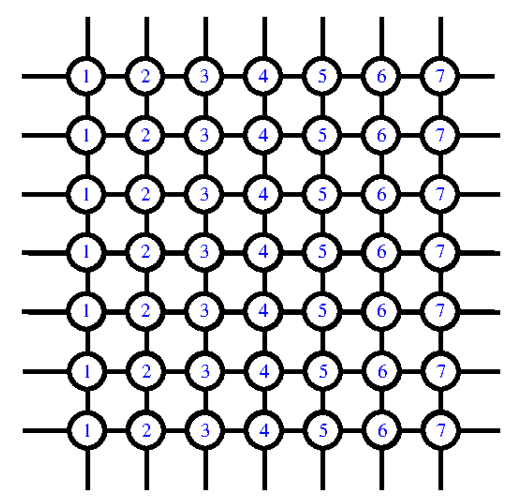

Figure 5. Order of computations for a single sweep of the grid.

If this process leads to the construction of a modified solution that serves a vertex that can not be reached with available edges, this vertex is removed from the solution. If the modified solution does not serve a vertex with $q_{V}>0$ that can be reached, this vertex must be added to the solution. One way to do so is to add it immediately after one of the nearest vertex that appears in the solution.

- Genetic algorithms : As with simulated annealing, we consider genetic algorithms whose solutions are ordered lists of vertices (that are split later). At each step, each node has a population of solutions. Two of these solutions can be mixed to obtain (generally two) children solutions. A larger population of children solutions is produced by this means, and the new population at the next step is taken as a subset of this children population, with a selection favoring the quality of the solutions, and their diversity. To share information between nodes, children solutions at node $\Xi^{(i, j)}$ can be created as mixes of two solutions at node $\Xi^{(i, j)}$, or of one solution at node $\Xi^{(i, j)}$ and one solution at a neighbouring nodes. As for simulated annealing, vertices may have to be added or removed to obtain a valid child solution. When an addition is needed, it can be done on the parent solution by adding the vertex immediately after a neighbouring vertex.

As we want to compute the actual collecting tour as a modification of one solution in this basis of solution, it makes sense to have several solutions available for each node, and to look for more robust solutions, which would need less alterations, and less drastic ones, to give a valid solution. In the literature, the main method to obtain robust solutions is to look for solution that perform the best against the worst intances of the parameters, with a min-max optimization (as is done in (Bertsimas and Sim, 2003)). There are several reasons we think this approach is not practical for our problem. First, it is hard to give a reliable upper bound on waste quantities. Even with a good global estimate of the quantity of waste produced, we can not exclude high local dicrepancies, with quantities much more important than expected in a given street. If reliable upper bounds could be given, they would be far superior to the expected values of waste quantities, and robust solutions based on worst case scenarios would be inefficient in many cases. Moreover, uncertainties on the availability of roads can not simply be managed by a worst case approach.

As a consequence, we give a few other paths to compute robust solutions. These solutions will not be as immunized to uncertainty as those constructed with robust optimization as defined in the literature, but they are designed to strike a compromise between robustness and efficiency.

To account for roads being less available than expected, one can take a higher threshold to include an edge in the problem at node $\Xi^{(i, j)}$, for example $\mathbf{P}\left[\rho_{e}=1 \mid \mathcal{F}_{1}\right]>0.75$, but there is a risk of creating vertices that can not be reached, thereby excluding them from the tour. Another way to mitigate the risk of road unavailability is to favor individaul vehicles visiting vertices that are close to each other. This can make rerouting a vehicle easier if an edge is no more available.

To account for the risk of waste quantities being locally more important than expected, one can limit vehicles to a fraction (for example $90 \%$, or $95 \%$ of their true capacity in the initial basis of solutions, to avoid having to change the list of vertices they are to visit (which happens when the total quantity of waste at these vertices exceeds the capacity of a vehicle). One can also compute solution where a small fraction of sites is visited twice, to have more leeway with vehicle capacities.

With genetic algorithms, and, in a more limited fashion, with ant colony solvers, it is possible to look for a set of diverse solutions for each node. This makes it more likely that one of these solutions can be easily adapted into a valid solution in the second phase.

\subsection{Derivation of actual solutions}

At time $t_{2}$, a large part of the uncertainty is removed as the collection tour is decided upon. The goal of our process is to be able to derive a valid collection tour using the basis of solutions constructed beforehand. To do this, one can reevaluate parameters $h$ and $\xi$ in light of the new information available, and consider solutions at nodes $\Xi^{(i, j)}$ near the reevaluated values. If one (or several) of these solutions are valid solutions of the problem at $t_{2}$, or can be easily adapted into valid solutions, one can take the best of these possibilities as the actual tour that is settled upon. What we call easy adaptations are:

- If an edge of the solution is unavailable, it is bypassed using a shortest path between the previous visited and the next visited node.

- If the nodes that are to be visited by a vehicle have a total quantity of waste that exceeds its capacity, one or more 
nodes are dropped of its schedule and reattributed to other vehicles that are still under their capacity.

Sungur et al. (Sungur et al., 2010) have devised a more sophisticated way to obtain a valid solution from a basis of solutions, with their "Master and Daily Scheduler" method. Before uncertainty is removed, they build a master solution, as the solution that can be best adapted on a fixed sample of scenarios. This adaptation is done by an insertion routine that adds new "customers" that need to be visited in a scenario but were not in the master solution. When a daily schedule needs to be produced, it is derived from the master solution, and then improved by a tabu search. While they work on a slightly different problem, their adaptation approach, combining an insertion routine and an improvement by tabu search, should also be tractable for our problem.

If it is not possible to find a valid solution by adapting the basis of solutions, we search for a valid solution of good quality by using again the same meta-heuristic algorithm. As the goal is not necessarily to find optimal solution, it is possible to use a quicker algorithm with fewer iterations. Solutions of the basis of solutions can be used as initial solutions (in a way that is similar to preconditioning for linear problem), with the reasoning that even if these solutions can not be used in full to produce a valid solution, they contain parts that could help build a good valid solution.

At time $t_{3}$, a solution is settled upon, and it is up to drivers to change their itinerary if one given road is not available, or head back to the depot once they have reached their full capacity. It is therefore useful to keep some robustness in the solution determined at $t_{2}$.

\section{CONCLUSIONS}

We have put forward a model for the progressive removal of the uncertainty, in the context of the computation of waste collection tours after a flood, the uncertain variables being waste quantities and road availability. With this model in mind, we have made two main contributions. We have described a method to find an efficient collection tour adapted to a particular situation in a constrained timeline, as there is generally little time between the time enough information is gathered to conceive a feasible tour and the time the waste collection should start. To do this, we proposed a method in three steps, the construction of a basis of solutions (based on the discretization of a low-dimensional set of random variables governing the behaviour of the final random variables), the computation of an actual solution by modification, using this basis, and the adaptation of the solution on the fly, when some variables are different from what was planned. We also proposed a framework to compute efficiently a set of solutions for the nodes of the grid in the space of random variable. This framework implies the simultaneous computation of solutions for every node of the grid, and the transmission of information between nodes. Such a transmission of information can be implemented using algorithms based on different meta-heuristic. We also described how to derive actual solutions from this basis, and how they can be adapted during the collection tour if necessary.

Much work remains to be done about the computation of the solutions on the grid in the space of random variables, first to prove that our method based on the sharing of information between nodes effectively accelerates the finding of optimal solutions, and then to study which algorithms, and which models for the communication of information lead to the most efficient method. Another subject for further research is the characterization of robust solutions, in the sense that they are more likely to lead to the finding of valid final solutions, and how algorithms can be adapted to find these robust solutions. Finally, the last improvement needed to make this framework practical is to define more possible modifications to adapt solutions and obtain a valid solution quickly, either during the computation of the actual solution, or even during the collection of the waste.

\section{REFERENCES}

Bányai, T., Tamás, P., Illés, B., Stankevičiūtè, Ž., Bányai, Á., 2019. Optimization of municipal waste collection routing: Impact of industry 4.0 technologies on environmental awareness and sustainability. International journal of environmental research and public health, 16(4), 634.

Ben-Tal, A., Nemirovski, A., 1998. Robust convex optimization. Mathematics of operations research, 23(4), 769-805.

Beraud, H., 2013. Initier la résilience du service de gestion des déchets aux catastrophes naturelles: le cas des territoires urbains et de l'inondation. PhD thesis, Université Paris-Est.

Beraud, H., Barroca, B., Jadot, J., Bauduceau, N., Hubert, G., 2012. Mécadépi. Méthode d'Evaluation et CAractérisation des DEchets Post Inondation. Rapport final. PhD thesis, Etablissement public Loire; FEDER.

Bertsimas, D. et al., 1988. The probabilistic vehicle routing problem.

Bertsimas, D., Sim, M., 2003. Robust discrete optimization and network flows. Mathematical programming, 98(1), 49-71.

Brown, C., Milke, M., Seville, E., 2011. Disaster waste management: A review article. Waste Management, 31, 1085-1098.

Campbell, A. M., Jones, P. C., 2011. Prepositioning supplies in preparation for disasters. European Journal of Operational Research, 209(2), 156-165.

Çelik, M., Ergun, Ö., Keskinocak, P., 2015. The post-disaster debris clearance problem under incomplete information. Operations Research, 63(1), 65-85.

El Ghaoui, L., Oustry, F., Lebret, H., 1998. Robust solutions to uncertain semidefinite programs. SIAM Journal on Optimization, 9(1), 33-52.

Ghanem, R., 1999. Ingredients for a general purpose stochastic finite elements implementation. Computer Methods in Applied Mechanics and Engineering, 168(1-4), 19-34.

Gruler, A., Fikar, C., Juan, A. A., Hirsch, P., Contreras-Bolton, C., 2017. Supporting multi-depot and stochastic waste collection management in clustered urban areas via simulationoptimization. Journal of simulation, 11(1), 11-19.

Hvattum, L. M., Løkketangen, A., Laporte, G., 2006. Solving a dynamic and stochastic vehicle routing problem with a sample scenario hedging heuristic. Transportation Science, 40(4), 421438. 
Jaillet, P., 1985. Probabilistic traveling salesman problems. PhD thesis, Massachusetts Institute of Technology.

Jaillet, P., 1988. A priori solution of a traveling salesman problem in which a random subset of the customers are visited. $O p$ erations research, 36(6), 929-936.

Keese, A., 2003. A review of recent developments in the numerical solution of stochastic partial differential equations (stochastic finite elements). Univ.-Bibl.

Laporte, G., 1992. The vehicle routing problem: An overview of exact and approximate algorithms. European journal of operational research, 59(3), 345-358.

Luther, L., 2010. Managing disaster debris: Overview of regulatory requirements, agency roles, and selected challenges. LIBRARY OF CONGRESS WASHINGTON DC CONGRESSIONAL RESEARCH SERVICE.

Mei, Y., Tang, K., Yao, X., 2010. Capacitated arc routing problem in uncertain environments. IEEE Congress on Evolutionary Computation, IEEE, 1-8.

Mete, H. O., Zabinsky, Z. B., 2010. Stochastic optimization of medical supply location and distribution in disaster management. International Journal of Production Economics, 126(1), 76-84.

Mladenović, N., Brimberg, J., Hansen, P., Moreno-Pérez, J. A., 2007. The p-median problem: A survey of metaheuristic approaches. European Journal of Operational Research, 179(3), 927-939.

Nobile, F., Tempone, R., 2009. Analysis and implementation issues for the numerical approximation of parabolic equations with random coefficients. International journal for numerical methods in engineering, 80(6-7), 979-1006.

Owen, S. H., Daskin, M. S., 1998. Strategic facility location: A review. European journal of operational research, 111(3), 423447.

Oyola, J., Arntzen, H., Woodruff, D. L., 2018. The stochastic vehicle routing problem, a literature review, part I: models. EURO Journal on Transportation and Logistics, 7(3), 193-221.

Rawls, C. G., Turnquist, M. A., 2010. Pre-positioning of emergency supplies for disaster response. Transportation research part B: Methodological, 44(4), 521-534.

Shen, Z., Dessouky, M. M., Ordóñez, F., 2009. A two-stage vehicle routing model for large-scale bioterrorism emergencies. Networks: An International Journal, 54(4), 255-269.

Sungur, I., Ren, Y., Ordóñez, F., Dessouky, M., Zhong, H., 2010. A model and algorithm for the courier delivery problem with uncertainty. Transportation science, 44(2), 193-205.

Tryoen, J., Le Maitre, O., Ndjinga, M., Ern, A., 2010. Intrusive Galerkin methods with upwinding for uncertain nonlinear hyperbolic systems. Journal of Computational Physics, 229(18), 6485-6511.

Wøhlk, S., 2008. A decade of capacitated arc routing. The vehicle routing problem: latest advances and new challenges, Springer, 29-48.

Yi, W., Özdamar, L., 2007. A dynamic logistics coordination model for evacuation and support in disaster response activities. European journal of operational research, 179(3), 1177-1193. 